\title{
Uncertainty propagation in Modern Physics Lab
}

\author{
George E. Hassel, ${ }^{1}$ Darren L. Broder, ${ }^{1,2}$ and John P. Cummings ${ }^{1}$ \\ ${ }^{1}$ Department of Physics \& Astronomy, Siena College, 515 Loudon Road, Loudonville, NY, 12211 \\ ${ }^{2}$ Taconic High School, 96 Valentine Road, Pittsfield, MA, 01201
}

\begin{abstract}
Uncertainty or error analysis is an important skill to be developed throughout the undergraduate physics laboratory curriculum. Numerical estimation and propagation of measurement uncertainties are a crucial part of interpreting and reporting laboratory results. The ability to clearly identify, explain and evaluate measurement uncertainty is also an important part of the broader goal of improving student writing and to modify poor writing habits. Modern Physics Lab provides a particularly opportune time to develop error propagation in detail. This paper examines uncertainty propagation in two example lab activities.
\end{abstract}

\section{INTRODUCTION}

Error analysis is recognized in two of the focus areas of the AAPT Recommendations for the Undergraduate Physics Laboratory Curriculum [1], and is stated as a primary learning objective in the Modern Physics Lab course at Siena College. The topic is first introduced in General Physics, then later reinforced in advanced laboratory courses in Mechanics, Electricity and Magnetism, and Experimental Techniques, as well as senior capstone research projects. In our curriculum, Modern Physics is a fall semester sophomore course that represents an opportune time to develop and emphasize these skills.

Error analysis is a required component of weekly lab reports, and is presented in a sequence similar to that described in the previous BFY Proceedings [2]. Lab reports are generally expected to include preliminary questions, data, sample calculations, results and conclusions. The mathematical level of error propagation requires the ability to compute partial derivatives at the level presented by Taylor's An Introduction to Error Analysis or similar texts [3].

Additional stated objectives for the lab include supplementing the abstract lecture material with hands-on experience and the continued development of graphing skills. The lab activities are further intended to develop a greater sense of experimental independence and to improve student writing habits.

\section{ERROR PROPAGATION IN EXAMPLE LABS}

The specific error analysis skills for most activities include:

1. make a numerical estimate in the uncertainty in measured values,

2. propagate the uncertainty in any calculations, e.g. the uncertainty in a speed is not the uncertainty in distance,

3. evaluate relative uncertainty for different variables, e.g. can the error in one or more measurements be ignored?,

4. properly report and interpret experimental results with uncertainty, using correct significant figures.

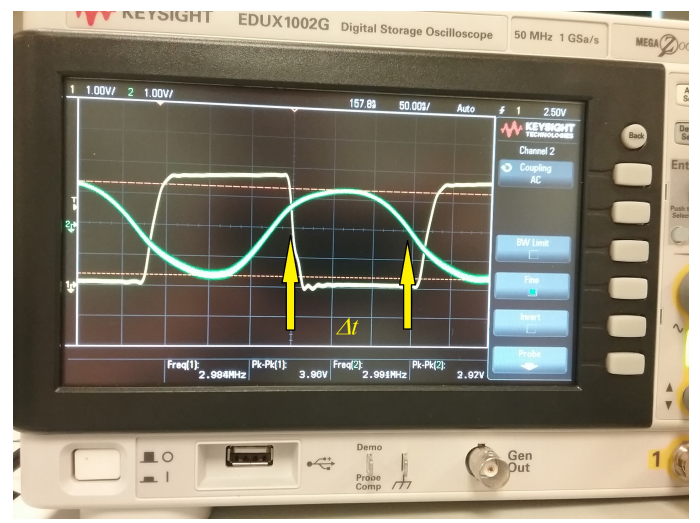

FIG. 1. Oscilloscope trace for speed of light experiment. Students must determine the time difference when the traces cross the central $\mathrm{x}$-axis (see arrows) between the yellow input square wave and the green sinusoidal detected wave. At $50 \mathrm{~ns} / \mathrm{div}$, this appears to be $27 / 8$ divisions, or $\Delta t=143.75 \mathrm{~ns}$, and the uncertainty can be estimated as the width of the trace, approximately $1 / 3$ of a minor division, or approximately $\delta(\Delta t)=4 \mathrm{~ns}$.

We examine the implementation of error analysis for two example lab activities that are likely used in many Modern Physics courses: speed of light (PASCO AP-8586) [4] and $\mathrm{e} / \mathrm{m}$ for the electron (TELTRON/ 3B Scientific U19155) [5].

\section{A. Speed of Light}

In this activity, the speed of light is determined by measuring $\Delta t$, the time for short laser pulses to travel a known path length, $\Delta x$, from the laser to a distant mirror, and back to a receiver. The time interval is measured as the difference between the function generator pulses used to drive the laser, and the signal received as shown in Figure 1. Delays introduced by the cables and electronics that comprise the apparatus add some additional phase shifting between the traces, and so it is not possible to calculate speed from one measurement of a direct ratio of $v=\Delta x / \Delta t$. If the delay is consistent, it is possible to determine the speed from the slope of a graph of several (8-10) data points. Sample data are included in Table I. 
TABLE I. Sample data of path length, $\Delta x$, and time interval, $\Delta t$, for speed of light. Negative time intervals are a result of instrumental delays and are physically meaningful

\begin{tabular}{cc}
\hline \hline$\Delta x(\mathbf{m})$ & $\Delta t(\mathbf{n s})$ \\
\hline $20.00 \pm 0.01$ & $40 \pm 5$ \\
$18.00 \pm 0.01$ & $30 \pm 5$ \\
$16.00 \pm 0.01$ & $25 \pm 5$ \\
$14.00 \pm 0.01$ & $20 \pm 5$ \\
$12.00 \pm 0.01$ & $15 \pm 5$ \\
$10.00 \pm 0.01$ & $5 \pm 5$ \\
$8.00 \pm 0.01$ & $0 \pm 5$ \\
$6.00 \pm 0.01$ & $-10 \pm 5$ \\
$4.00 \pm 0.01$ & $-15 \pm 5$ \\
$2.00 \pm 0.01$ & $-20 \pm 5$ \\
\hline \hline
\end{tabular}

The oscilloscope traces can be somewhat blurry, especially in the case of the detected wave. The uncertainty associated with this blur can be estimated as $\delta(\Delta t)=4 \mathrm{~ns}$ for the measurement shown in Figure 1, and a range of estimates may be considered acceptable. This time interval was measured for a path length of $\Delta x=7 \mathrm{~m}$, over which it may be reasonable to estimate an uncertainty of $\delta(\Delta x)=1 \mathrm{~cm}$ resulting from the placement of the meter stick. The speed calculated from this single data point, $v=\Delta x / \Delta t \sim 5 \times 10^{7} \mathrm{~m} / \mathrm{s}$ is not accurate because of the inherent delay.

In this experiment, the time interval is the dependent variable and is associated with significantly larger measurement uncertainty. It is thus preferable to determine the slope of a plot of $\Delta t$ vs. $\Delta x$ by linear regression analysis. The inverse of that slope is the experimental result for the speed of light, with a value of $v=2.98 \times 10^{8} \mathrm{~m} / \mathrm{s}$ and uncertainty $\delta v \sim 8 \times 10^{6} \mathrm{~m} / \mathrm{s}$ for the data in Table I. The coefficients of linear fit, associated uncertainties, and correlation coefficient may be calculated by statistical functions such as LINEST in Excel. The least squares fitting method described in Chapters 8 and 9 of Taylor's book provide additional detail that make this calculation accessible to sophomore level students. Further, it is possible to evaluate the uncertainty associated with the measurement of $\Delta t$ from Equation 8.14 of Taylor for deviations from the statistical fit. For this data, we calculate $\sigma_{\Delta t}=1.7 \mathrm{~ns}$ associated with the fit. This value is approximately $1 / 3$ of the uncertainty associated with blurring of $5 \mathrm{~ns}$ in Table I, consistent with a factor of $\sqrt{N}$ for this data set.

It may be desirable to estimate the slope and uncertainty with a less rigorous method. One option is to plot the data points with error bars, and to sketch additional fits that reasonably cross through the error bars to estimate the possible range of slopes. This can also be accomplished by choosing two points in the data set, and drawing a fit to those. Using the first and last points in Table I, the result is $v=3.0 \pm 0.6 \times 10^{8}$ $\mathrm{m} / \mathrm{s}$. This method yields a similar slope with a substantially larger uncertainty, but may help students to visually compare the uncertainty in the slope with the error bars.

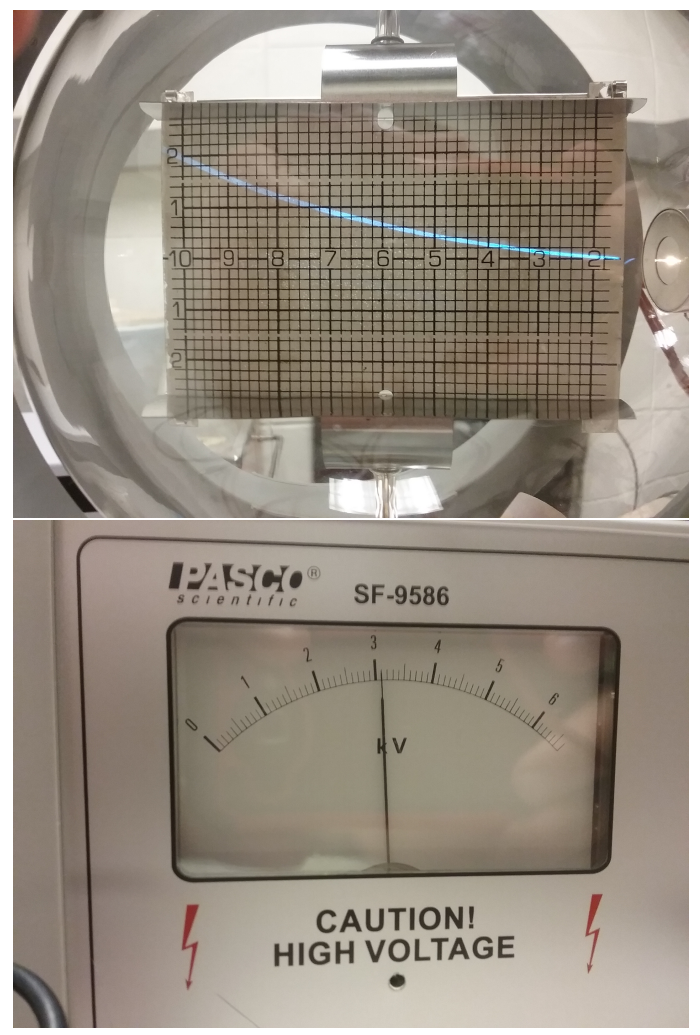

FIG. 2. Images of electron beam position (top) and accelerating voltage (bottom) for the $e / m$ ratio experiment. Students adjust accelerating voltage and coil current to get the beam to cross the $(10 \mathrm{~cm}$, $2 \mathrm{~cm}$ ) grid coordinate.

\section{B. $e / m$ for the electron}

In this activity, students determine the fundamental charge to mass ratio of $e / m$ for the electron using Helmholtz coils to deflect a fluorescent electron beam generated within a cathode ray tube. The ratio is:

$$
\left(\frac{e}{m}\right)=\frac{2 V_{a}}{B^{2} r^{2}}
$$

where $V_{a}$ is the accelerating voltage in the tube, $B$ is the magnetic field between coils:

$$
B=\frac{8 \mu_{0} N I}{a \sqrt{125}},
$$

where $N$ is the number of coils, $I$ is the current through the coils, a is the coil radius, and $\mu_{0}$ is the magnetic permeability. The radius of curvature of the beam, $r$, is:

$$
r=\frac{x^{2}+y^{2}}{2 y},
$$

where $x$ and $y$ are the horizontal and vertical coordinates of a point along the beam as illustrated in Figure 2.

In this experiment, students set an accelerating voltage, $V_{a}$, to see the beam, and then adjust the current, $I$, until the beam 
crosses the upper or lower-most corner of a printed grid. Unlike the speed of light experiment, it is possible to determine the $(\mathrm{e} / \mathrm{m})$ ratio from one pair of $V_{a}, I$ measurements. However, it is preferable to measure a range of values for $V_{a}$ and $I$ that successfully deflect the beam to the same position.

TABLE II. Sample data for $e / m,(x, y)=(10 \mathrm{~cm}, 2 \mathrm{~cm})$, other constants from manual [5]: $N=320, a=0.068 \mathrm{~m}$.

\begin{tabular}{cccc}
\hline \hline$V_{a}(\mathbf{k V})$ & $I(\mathbf{A})$ & $B(\mathbf{m T})$ & $e / m(\mathbf{C} / \mathbf{k g})$ \\
\hline 2.5 & 0.155 & 0.656 & $1.72 \times 10^{11}$ \\
3.0 & 0.184 & 0.778 & $1.46 \times 10^{11}$ \\
3.5 & 0.197 & 0.833 & $1.49 \times 10^{11}$ \\
4.0 & 0.217 & 0.918 & $1.40 \times 10^{11}$ \\
4.5 & 0.239 & 1.0 & $1.30 \times 10^{11}$ \\
\hline \hline
\end{tabular}

There are multiple ways to determine the experimental uncertainty for this activity. Since the beam is deflected to the same gridpoint, all measured combinations of $V_{a}$ and $I$ should yield the same $(\mathrm{e} / \mathrm{m})$ ratio. Thus, it is appropriate to find a mean value for all of the experimental trials and to determine the uncertainty from standard deviation of the mean for $N$ trials:

$$
\sigma_{\overline{(e / m)}}=\frac{\sigma_{(e / m)}}{\sqrt{N}} .
$$

For the data in Table II, the mean value is $e / m=1.47 \times$ $10^{11} \mathrm{C} / \mathrm{kg}$ with a standard error of $7 \times 10^{9} \mathrm{C} / \mathrm{kg}$.

Alternatively, it is possible to propagate the uncertainty by the more general form of the quadrature formula. There are three independent variables represented in the formula for $(e / m): r, B$, and $V_{a}$. The result for the propagation in quadrature of $\delta(e / m)$ is:

$$
\sqrt{\left(\frac{\partial\left(\frac{e}{m}\right)}{\partial V_{a}} \delta V_{a}\right)^{2}+\left(\frac{\partial\left(\frac{e}{m}\right)}{\partial B} \delta B\right)^{2}+\left(\frac{\partial\left(\frac{e}{m}\right)}{\partial r} \delta r\right)^{2}} .
$$

This method represents a more challenging application of partial derivatives, where the individual terms can be expressed as:

$$
\begin{aligned}
& \left|\frac{\partial\left(\frac{e}{m}\right)}{\partial V_{a}}\right|=\frac{2}{B^{2} r^{2}}, \\
& \left|\frac{\partial\left(\frac{e}{m}\right)}{\partial B}\right|=\frac{4 V_{a}}{B^{3} r^{2}},
\end{aligned}
$$

and

$$
\left|\frac{\partial\left(\frac{e}{m}\right)}{\partial r}\right|=\frac{4 V_{a}}{B^{2} r^{3}}
$$

The radius $r$ and the magnetic field $B$ are functions of measured quantities in position and current, $x, y$, and $I$. The uncertainties $\delta x, \delta y$ arise from blurring of the beam, and $\delta V_{a}$ from the position of the needle. The uncertainty in magnetic field is directly related to the uncertainty in current, $\delta I$, which can be estimated from instrument uncertainty in the ammeter used. The uncertainty in magnetic field $\delta B=C \delta I$, for $C=4.2 \times 10^{-3} \mathrm{~T} / \mathrm{A}$ for the coils used [5]. The uncertainty in radius of curvature is

$$
\delta r=\sqrt{\left(\frac{x}{y} \delta x\right)^{2}+\left(\frac{y^{2}-x^{2}}{2 y^{2}} \delta y\right)^{2}} .
$$

For measurement uncertainty estimates of $\delta V_{a} \sim 25 \mathrm{~V}$, $\delta I \sim 0.001 \mathrm{~A}, \delta x \sim \delta y \sim 1 \mathrm{~mm}$ and the data in Table II, the propagated uncertainty ranges from $\delta(e / m) \sim$ $1.4-1.7 \times 10^{10} \mathrm{C} / \mathrm{kg}$, a factor of approximately $\sqrt{N}$ larger than the statistical standard error of Equation 4. This is reasonable, as this method determines a propagated uncertainty for individual measurements, and the statistical approach represents the mean.

A possible simpler approach involves adding the relative errors of $V_{A}, I, x$, and $y$ in quadrature. For the data provided, the result of this is $\delta(e / m) \sim 7-9 \times 10^{9} \mathrm{C} / \mathrm{kg}$. Although this method is not as rigorous, it provides a reasonable estimate in this case, and may be easier for students to utilize.

\section{IMPROVING STUDENT HABITS}

Modern Physics Lab is one of the first courses in which our students encounter error analysis in detail as a primary objective. Students come from a variety of backgrounds, and have writing habits that have developed over time. A major unstated objective of our Modern Physics Lab course is to improve several student habits that affect report writing, particularly with respect to error analysis and interpreting results. This discussion is anecdotal rather than a controlled survey [6], however, we identify some similar issues.

A very common habit is to use the blanket term "human error" to discuss measurement uncertainty, without specifically identifying or making a numerical estimate of uncertainty. This habit is rooted in confusion about the meaning of the word "error" as necessarily referring to mistakes in an experiment [6]. Different lab instructors will have different preferences regarding terminology, but the key is to emphasize the estimation and propagation of numerical values for uncertainty. Holmes and Wieman further explain that the use of percent error rather than a more careful consideration of uncertainty when interpreting results can add to this confusion. Usage of the specific terminology of Type A uncertainty evaluation, associated with the statistics of repeated measurements, and Type B, associated with estimation or assumption of uncertainty, may help to clarify the treatment of uncertainty [7]. At the time of this writing, this terminology has not yet been formally adopted into our course. Most of our 
treatment involves Type B. The first week is devoted to measurement activities that illustrate the need for propagation.

Another common habit is to include some version of the statement "Overall, this lab was a great success and I learned a lot" as a conclusion, regardless of the quality of the results. Emphasis on a detailed description of the quantitative results, numerical description of error, and a sample conclusion can help to eliminate this habit.

Formula hunting, or rapidly choosing a formula without understanding the meaning, is a habit that students commonly use in both lab and lecture. This habit can present a challenge when students fail to understand that there is not one single formula such as percent error that is appropriate and applicable in all situations. The general formula for propagation in quadrature is often applicable, but requires a careful consideration of the experimental conditions and skill with partial derivatives. The notation can appear formidable to sophomore-level students, particularly in determining which term represents which quantity. These concerns can be alleviated with worked examples and an emphasis on sequentially building data analysis skills [2].

A further goal of the lab course is to develop a greater sense of independence on the part of the student. Students are often conditioned to ask "is this correct?" in reference to data, results and interpretation at various stages of conducting an experiment and writing a report. Increasing safety and supervision standards require a greater instructor presence in the lab, which can lead to a greater frequency and persistence of such questions. A somewhat related, common complaint is that lab activities may precede or supersede lecture coverage of course material. This course uses "canned" activities with known results rather than open-ended projects to help develop student ability to prepare and to self-evaluate. Prelab preparation such as required preliminary questions, redirected questioning, such as "do you think it's correct, and why?", and the estimation of measurement uncertainty may help students to identify and improve lab techniques and interpretation skills, and to ask themselves "is this approach, measurement, or result reasonable?" A common example is that students often forget that the path length $\Delta x$ in the speed of light lab is double the laser-mirror distance, and thus find a result that is almost exactly half of the expected value of $v \sim 3 \times 10^{8} \mathrm{~m} / \mathrm{s}$. They can often self-identify this mistake with group discussions or instructor's suggestion.

\section{CONCLUSIONS}

Error analysis is recognized as an important undergraduate physics laboratory skill, particularly as described in the $A A P T$ Recommendations. Students often have difficulty understanding and applying uncertainty in data analysis and interpretation, often because of a lack of exposure or because of a perceived need to obtain a "correct" answer. As a result, students often view error analysis as a secondary concern, and rush through or ignore it. It is important to introduce and develop aspects of error analysis throughout the curriculum.

We have outlined strategies for implementing error analysis, both estimation of uncertainty and propagation to a result, in two example Modern Physics Lab activities. The estimation method is most similar to Type B [7] for both examples. In each case, we have identified a simpler approach, as well as a more rigorous one. Notes and rubrics for other lab activities are available to share upon request.

As an instructor, it can be difficult to distinguish between activities that challenge and develop skills in error analysis, and those that overwhelm student abilities. The course described in this paper is mostly populated with sophomore Physics majors and minors with a co-requisite of Multivariable Calculus. Lab activities that reinforce skills introduced in General Physics such as graphing, estimates of error bars, average, and standard deviation are appropriate. This course is an excellent opportunity to sequentially introduce the estimation of uncertainty and associated statistics, error propagation involving partial derivatives, and to emphasize writing conclusions that accurately reflect on the numerical results obtained.

\section{ACKNOWLEDGMENTS}

The authors gratefully acknowledge the many contributions of Prof. Josh Diamond to the Siena College community, and especially to the development of meaningful activities in the Modern Physics lab. His contagious enthusiasm for physics, approachability, and warm demeanor earned the respect and admiration of many colleagues, students and friends.
[1] J. Kozminski, et al. AAPT Recommendations for the Undergraduate Laboratory Curriculum https://www.aapt.org/Resources/ upload/LabGuidelinesDocument_EBendorsed_nov10.pdf (November 2014).

[2] T. Roach, in 2015 BFY Proceedings, edited by Eblen-Zayas, Behringer, and Kozminski (2015), p. 88.

[3] J.R. Taylor, An Introduction to Error Analysis: The Study of Uncertainities in Physical Measurements (University Science
Books, CA, 1982).

[4] https://www.pasco.com/file_downloads/Downloads_Manuals/ Laser-Speed-of-Light-Apparatus-AP-8586.pdf

[5] https://www.a3bs.com/product-manual/1000651_EN.pdf

[6] N.G. Holmes and C.E Wieman, in 2015 BFY Proceedings, edited by Eblen-Zayas, Behringer, and Kozminski (2015), p. 44.

[7] https://physics.nist.gov/cuu/Uncertainty/basic.html 